\title{
Presence of systemic autoimmune disorders in patients with autoimmune thyroid diseases
}

\author{
M G Tektonidou, M Anapliotou, P Vlachoyiannopoulos, H M Moutsopoulos
}

Ann Rheum Dis 2004;63:1159-1161. doi: 10.1136/ard.2004.022624

\begin{abstract}
Objective: To evaluate the prevalence of antinuclear antibodies (ANA) in patients with autoimmune thyroid diseases (ATD) and the presence of systemic autoimmune disorders among ANA positive patients with ATD.

Methods: 168 consecutive patients with ATD with positive antithyroid antibodies and 75 healthy subjects were tested for the presence of ANA. ANA positive patients were further evaluated by complete history, physical examination, blood and urine tests, and immunological studies. Patients with subjective xerophthalmia and xerostomia were examined by objective tests.

Results: $58 / 168$ (35\%) patients with ATD were ANA positive compared with $7 / 75(9 \%)$ healthy controls $(p=0.001)$. Of 58 ANA positive patients, 6 (10\%) had anti-Ro antibodies, 1 had anti-Ro and anti-La antibodies, 7 (12\%) had anti-dsDNA antibodies, and 7 (12\%) had medium levels of lgG and/or IgM anticardiolipin antibodies (aCL). No healthy subjects had positive anti-dsDNA, antibodies against the extractable nuclear antigens, or aCL. 5/58 (9\%) patients fulfilled the criteria for Siögren's syndrome (SS). Two patients had features related to systemic lupus erythematosus. No healthy subjects had clinical or laboratory characteristics of systemic autoimmune disorders.

Conclusion: ANA are detected in 1/3 of patients with ATD. Anti-dsDNA, anti-Ro, and aCL can also be found in ANA positive patients with ATD. SS occurs in about $1 / 10$ of ANA positive patients with ATD.
\end{abstract}

\section{A} utoimmune thyroid diseases (ATD)-namely, Hashimoto's thyroiditis and Graves' disease, are organ-specific autoimmune disorders characterised by the presence of antibodies against the thyroglobulin, thyroid peroxidase, or thyrotropin receptor autoantigens. ${ }^{1}$ The existence of ATD among patients with systemic autoimmune diseases such as systemic lupus erythematosus, Sjögren's syndrome (SS), or rheumatoid arthritis has been well recognised..$^{2-8}$ On the other hand, antinuclear antibodies (ANA) have been frequently detected in patients with ATD with uncertain clinical significance. ${ }^{9-11}$ Other autoantibodies associated with systemic autoimmune diseases such as antidsDNA antibodies and antibodies against the extractable nuclear antigens have been sporadically mentioned. ${ }^{7-12}$ However, the occurrence of systemic autoimmune diseases among patients with ATD remains unclear.

The purpose of this study was to evaluate the prevalence of ANA in the sera of 168 consecutive patients with ATD and the existence of systemic autoimmune diseases among ANA positive patients by clinical and laboratory examinations.

\section{PATIENTS AND METHODS}

In this study were included 168 consecutive patients with ATD (25 male, 143 female) with positive antiperoxidase and/ or antithyroglobulin antibodies and 75 healthy volunteers matched for age and sex. One hundred and twenty patients had Hashimoto's thyroiditis and 48 had Graves' disease. The diagnosis of ATD was made according to established criteria. ${ }^{1}$ None of the above patients had a history of systemic autoimmune disorders.

The above subjects were first examined for the presence of ANA in their sera. ANA positive subjects were further evaluated by a complete history and physical examination, blood tests (packed cell volume, white blood cells, platelets, erythrocyte sedimentation rate, creatinine, aspartate aminotransferase, alanine aminotransferase, $\gamma$-glutamyltransferase, alkaline phosphatase), urine tests as well as immunological studies for other more specific autoantibodies such as antidsDNA, antibodies against the extractable nuclear antigens (Ro/SSA, La/SSB, RNP, Sm), and anticardiolipin antibodies (aCL). Patients with subjective xerophthalmia and/or xerostomia assessed by questionnaire were further examined for the presence of keratoconjunctivitis sicca by Schirmer's test and rose bengal staining and for xerostomia by sialometry and minor salivary gland biopsy. ${ }^{13}$

The diagnosis of systemic autoimmune disorders was based on American Rheumatism Association criteria. SS was diagnosed according to criteria suggested by the American-European Consensus Group. ${ }^{13}$ Informed consent and ethical committee approval were received.

\section{Immunological studies}

ANA were determined by indirect immunofluorescence using Hep-2 cells as substrate, anti-dsDNA antibodies by enzyme linked immunosorbent assay (ELISA; normal range 0-7 IU), antibodies against the extractable nuclear antigens by counterimmunoelectrophoresis, IgG and IgM aCL by enzyme immunosorbent assay (normal range $0-100 \mathrm{IU}$ ). ${ }^{14}$ In the above studies commercial kits were not used but "home made" tests for the measurement of autoantibodies.

\section{RESULTS}

The mean (SD) age of the patients was 51.2 (13.2) years and of the controls 49.8 (12.3) years. The mean disease duration was $6.3(2.3)$ years.

ANA positivity was found in 58/168 (35\%) patients with ATD compared with $7 / 75(9 \%)$ healthy controls $(p=0.001)$. Fifty two of the above 58 patients (39 with Hashimoto's thyroiditis and 13 with Graves' disease) were receiving treatment with thyroxine, carbimazole, or a combination of these. Among the 58 ANA positive patients, six (10\%) had anti-Ro antibodies, one had anti-Ro and anti-La antibodies, seven (12\%) had anti-dsDNA antibodies, and seven (12\%) had aCL (five had IgG aCL and two had IgM aCL). The mean (SD) level of anti-DNA antibodies was 13.8 (3.2) IU (range

Abbreviations: $\mathrm{aCL}$, anticardiolipin antibodies; $\mathrm{ANA}$, antinuclear antibodies; ATD, autoimmune thyroid diseases; ELISA, enzyme linked immunosorbent assay; SS, Siögren's syndrome 
12-21). The mean (SD) titres of IgG and IgM aCL were 145 (13.5) IU (range 135-168) and 158 (16.5) IU (range 140183), respectively.

Two patients had leucopenia (white blood cells $<4 \times 10^{9} / 1$ ), but none had lymphopenia. No patient had abnormal liver enzymes, serum creatinine levels, and urine tests.

None of the healthy subjects had positive anti-dsDNA, antibodies against the extractable nuclear antigens, aCL, or other abnormal serological examinations.

\section{Systemic autoimmune diseases in ANA positive patients}

Seven patients had subjective xerophthalmia and six subjective xerostomia. Keratoconjunctivitis sicca and xerostomia were found by objective tests in five and four patients, respectively. Five patients fulfilled the criteria for SS. All the above five patients had positive minor salivary gland biopsies.

Two patients had SLE-like features: one of them had arthralgias, leucopenia, positive anti-dsDNA antibodies; and the other had arthralgias, mouth ulcers, livedo reticularis, anti-dsDNA antibodies, and mean levels of IgG aCL. Four patients had Raynaud's phenomenon and seven had arthralgias but without signs or symptoms of systemic autoimmune diseases. None of the seven patients with increased IgG and/ or IgM aCL had clinical manifestations of antiphospholipid syndrome, such as arterial or venous thromboses or recurrent abortions.

In addition, none of the healthy subjects had clinical or laboratory findings related to systemic autoimmune disorders.

\section{DISCUSSION}

The results of this study showed a high prevalence of ANA in patients with ATD compared with healthy controls $(p=0.001)$. Other non-thyroid autoantibodies such as antidsDNA, anti-Ro antibodies, and aCL were also detected. Furthermore, clinical and serological markers of systemic autoimmunity were found in ANA positive patients.

An increased prevalence of ANA among patients with ATD has been well recognised. ${ }^{9-11}$ However, the existence of other non-thyroid autoantibodies in the above patients remains controversial. The conflicting results concerning the presence of anti-dsDNA antibodies in ATD can probably be explained by the different diagnostic assays which were used in earlier studies. ${ }^{9101215}$ These studies reported positive anti-dsDNA antibodies when measured by radioimmunoasssay but negative antibodies when the more specific Crithidia luciliae assay was used..$^{10} 12$ Serum antibodies against the Ro/SSA and La/SSB antigens have been only sporadically described in ATD. Hansen et al reported that $1 / 63$ patients with ATD had precipitating anti-Ro antibodies and none had precipitating anti-La antibodies. ${ }^{7}$ When the latter antibodies were tested by ELISA, 17/63 sera had abnormal values but in low titres. Other studies examining for the presence of several nonthyroid antibodies in ATD had not included anti-Ro and antiLa antibodies or they failed to detect them. ${ }^{10}{ }^{11}$ The presence of aCL has been rarely reported in patients with ATD. Paggi and coworkers detected IgG and/or IgM aCL in 17/31 patients with ATD. ${ }^{16}$ Osundeko et al found increased levels of IgG and IgM aCL in Hashimoto's thyroiditis. ${ }^{17}$ However, other authors reported negative aCL in patients with Hashimoto's thyroiditis or Graves' disease. ${ }^{11}$

Despite the existence of the above autoantibodies, few publications have studied the occurrence of systemic autoimmune diseases in patients with ATD. Williamson et al found no difference in the frequency of positive rose bengal staining and parotid scintigraphy findings between patients with ATD and controls. ${ }^{18}$ In the study by Hansen et al, 19/63 enrolled patients were tested for xerostomia by sialometry and/or lip biopsy, and for keratoconjunctivitis sicca by three objective tests. ${ }^{7}$ Six of them had keratoconjunctivitis sicca together with xerostomia, while two of the above six had autoimmune sialadenitis in lip biopsies. Coll et al studied the prevalence of xerostomia and keratoconjunctivitis sicca in 176 asymptomatic patients with ATD. ${ }^{19}$ Nineteen of 52 patients tested for xerostomia by salivary scintigraphy and/ or gland biopsy and 9/170 patients examined for keratoconjunctivitis sicca by Schirmer and rose bengal tests had positive results. Anti-Ro and anti-La antibodies were not measured. The authors reported that features of SS were diagnosed in $24 \%$ of the patients. SS was defined by the presence of keratoconjunctivitis sicca and/or xerostomia. Warfvinge et al found that 11/19 cases with autoimmune thyroiditis had various degrees of salivary gland involvement on the basis of lip biopsy analysis, scintigraphy of parotids, and/or sialometry. ${ }^{20}$

In the above studies the diagnosis of SS was based on the results of several objective tests for salivary gland or ocular involvement. In our study we used the American-European classification criteria for SS, which included subjective and objective tests for salivary gland and ocular involvement as well as measurement of serum autoantibodies (ANA, anti-Ro or anti-La, rheumatoid factor). ${ }^{13}$

As far as we know, this is the first study investigating any incidence of systemic autoimmune diseases among ANA positive patients with ATD by complete history, physical examination, serological and immunological tests, and objective tests for keratoconjunctivitis sicca and xerostomia. We found a $9 \%$ prevalence of SS in patients with ATD with ANA compared with an estimated prevalence of $0.6 \%$ in a closed rural community in Greece. ${ }^{21}$ Two cases with SLE-like features were also documented.

We conclude that SS occurs in about 1/10 of ANA positive patients with ATD, suggesting that common pathogenetic mechanisms have a role in the development of thyroid and salivary gland immune diseases. A polyclonal immune response against organ-specific and non organ-specific autoantigens may exist in patients with ATD. The autoimmune response to the thyroid follicular cells can also spread to the salivary gland epithelium..$^{22}$ Clinicians should be alert to symptoms and signs of systemic autoimmune diseases, especially SS, in ANA positive patients with ATD.

\section{Authors' affiliations}

M G Tektonidou, M Anapliotou, P Vlachoyiannopoulos,

H M Moutsopoulos, Department of Pathophysiology, Medical School, National University of Athens, Athens, Greece

Correspondence to: Dr M G Tektonidou, Department of Pathophysiology, Medical School, National University of Athens, 75 Mikras Asias Str, 11527 Athens, Greece; balts@otenet.gr

Accepted 19 April 2004

\section{REFERENCES}

1 Weetman AP, McGregor AM. Autoimmnune thyroid disease: further developments in our understanding. Endocr Rev 1994;15:788-830.

2 Pyne D, Isenberg DA. Autoimmune thyroid disease in systemic lupus erythematosus. Ann Rheum Dis 2002;61:70-2.

3 Weetman AP, Walport MJ. The association of autoimmune thyroiditis with systemic lupus erythematosus. Br J Rheumatol 1987;26:359-61.

4 Karsh J, Pavlidis N, Weintraub BD, Moutsopoulos HM. Thyroid disease in Sjögren's syndrome. Arthritis Rheum 1980;23:1326-9.

5 Perez B, Kraus A, Lopez G, Cifuentes M, Alarcon-Segovia D. Autoimmune thyroid disease in primary Siögren's syndrome. Am J Med 1995;99:480-4.

6 Shiroky JB, Cohen M, Ballachey ML, Neville C. Thyroid dysfunction in rheumatoid arthritis: a controlled prospective survey. Ann Rheum Dis 1993;52:454-6.

7 Hansen BU, Ericsson UB, Henricsson V, Larsson A, Manthorpe R, Warfvinge $G$. Autoimmune thyroiditis and primary Sjögren's syndrome: 
clinical and laboratory evidence of the coexistence of the two diseases. Clin Exp Rheumatol 1991;9:137-41

8 Scofield RH. Autoimmune thyroid disease in systemic lupus erythematosus and Sjögren's syndrome. Clin Exp Rheumatol 1996;14:321-30.

9 Katakura M, Yamada T, Aizawa T, Hiramitsu K, Yukimura Y, Ishihara M, et al. Presence of antideoxyribonucleic acid antibody in patients with hyperthyroidism of Grave's disease. J Clin Endocrinol Metab 1987;64:405-8.

10 Baethge BA, Levine SN, Wolf RE. Antibodies to nuclear antigens in Graves'disease. J Clin Endocrinol Metab 1988;66:485-8.

11 Petri M, Karlson EW, Cooper DS, Ladenson PW. Autoantibody tests in autoimmune thyroid disease: a case-control study. I Rheumatol $1991 ; 18: 1529-31$.

12 McDermott MT, West SG, Emlen JW, Kidd GS. Antideoxyribonucleic acid antibodies in Grave's disease. $J$ Clin Endocrinol Metab 1990;71:509-11.

13 Vitali C, Bombardieri S, Jonsson R, Moutsopoulos HM, Alexander EL, Carsons SE, et al. Classification criteria for Sïgren's syndrome: a revised version of the European Criteria proprosed by the American-European Consensus group. Ann Rheum Dis 2002;61:554-8.

14 Tektonidou MG, loannidis JP, Boki KA, Vlachoyiannopoulos PG, Moutsopoulos HM. Prognostic factors and clustering of serious clinical outcomes in antiphospholipid syndrome. Q J Med 2000;93:523-30.
15 Loviselli A, Velluzzi F, Pala R, Marcello A, Nurchis P, Mathieu A, et al. Circulating antibodies to DNA-related antigens in patients with autoimmune thyroid disorders. Autoimmunity 1992;14:33-6.

16 Paggi A, Caccavo D, Ferri GM, Di Prima MA, Amoroso A, Vaccaro F, et al. Anti-cardiolipin antibodies in autoimmune thyroid diseases. Clin Endocrinol 1994;40:329-33

17 Osundeko O, Hasinski S, Rose LI. Anticardiolipin antibodies in Hashimoto's disease. Endocr Pract 2001;7:181-3.

18 Williamson J, Cant JS, Mason DK, Greig WR, Boyle JA. Siögren's syndrome and thyroid disease. Br J Ophthalmol 1967;51:721-6.

19 Coll J, Anglada J, Tomas S, Reth P, Goday A, Millan M, et al. High prevalence of subclinical Sjögren's syndrome features in patients with autoimmune thyroid disease. J Rheumatol 1997;24:1719-24.

20 Warfvinge G, Larsson A, Henricsson V, Ericsson UB, Hansen B, Manthorpe R. Salivary gland involvement in autoimmune thyroiditis, with special reference to the degree of association with Sjögren's syndrome. Oral Surg Oral Med Oral Pathol 1992;74:288-93.

21 Dafni UG, Tzioufas AG, Staikos P, Skopouli FN, Moutsopoulos HM. Prevalence of Sjögren's syndrome in a closed rural community. Ann Rheum Dis 1997; $56: 521-5$.

22 Moutsopoulos HM. Sïgren's syndrome: autoimmune epithelitis. Clin Immunol Immunopathol 1994;72:162-5. 